\title{
Improving Robotic Cooking using Batch Bayesian Optimization
}

\author{
Kai Junge ${ }^{1}$, Josie Hughes ${ }^{1}$, Thomas George Thuruthel $^{1}$ and Fumiya Iida ${ }^{1}$
}

\begin{abstract}
With advances in the field of robotic manipulation, sensing and machine learning, robotic chefs are expected to become prevalent in our kitchens and restaurants. Robotic chefs are envisioned to replicate human skills in order to reduce the burden of the cooking process. However, the potential of robots as a means to enhance the dining experience is unrecognised. This work introduces the concept of food quality optimization and its challenges with an automated omelette cooking robotic system. The design and control of the robotic system that uses general kitchen tools is presented first. Next, we investigate new optimization strategies for improving subjective food quality rating, a problem challenging because of the qualitative nature of the objective and strongly constrained number of function evaluations possible. Our results show that through appropriate design of the optimization routine using Batch Bayesian Optimization, improvements in the subjective evaluation of food quality can be achieved reliably, with very few trials and with the ability for bulk optimization. This study paves the way towards a broader vision of personalized food for taste-and-nutrition and transferable recipes.
\end{abstract}

\section{INTRODUCTION}

Robotic preparation of cooked food is a challenging task. The progression towards a fully automated robotic chef involves solving hard problems in robot manipulation, computer vision, tactile sensing, and human-robot-interaction [1]. Consequently, current demonstrations of robotic chefs are inferior in their capabilities compared to their biological counterparts. However, robotic technologies have the unique ability to accurately and precisely vary their actions and store retrievable quantitative information. This provides a framework to create consistent, parameterizable and controllable cuisines.

Automation in the food industry is a rapidly growing field. This has introduced faster, reliable and cheaper techniques for quality inspection [2], [3], [4], processing [5], handling [6], [7], and packaging. Unlike industrial food handling, kitchen robots are expected to be adaptable with the ability to cook numerous recipes, handle multiple existing kitchen tools and conform to the user's subjective preferences. A good example of this is the world's first robotic kitchen developed by the Moley Robotics (https://www.moley.com/).

Previous researches on robotic chefs or automated cooking focus on different aspects of the cooking process. Bollini et.al. investigated sensing and manipulation strategies for a robot that bakes cookies [8] and natural-language processing techniques for recipe interpretation [9]. A similar work on

\footnotetext{
${ }^{1}$ Kai Junge, Thomas George Thuruthel and Fumiya Iida are with the Bio-Inspired Robotics Lab, Department of Engineering, University of Cambridge, UK. kcj21@cam.ac.uk, jaeh2@cam.ac.uk, tg444@cam.ac.uk, fi224@cam.ac.uk

Source code available at https://github.com/kaijunge/ OmeletteBot
}

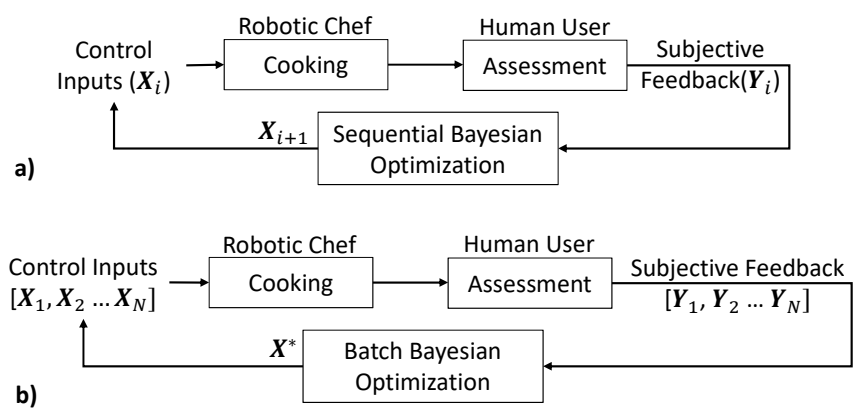

Fig. 1. The two optimization approaches that we investigate in this paper for food optimization. The main difference among two is the protocol for obtaining the qualitative data.

generating action plans from online instructions was done using a pancake making robot [10]. Other works involve tracking the state of the cooking ingredient for advanced manipulation tasks, as required for a pizza making robot [11]. All these studies strive towards the aim of replicating the dexterity and intelligence of a human chef. Alternatively, researchers are looking into non-traditional ways of food manufacturing based on 3D food printing [12]. Such technologies would allow users to create food products that are highly customizable and makes them an appealing solution for creating nutritious and visually appealing food products [13]. However, they are limited in the type of ingredients they can print and does not involve the process of cooking.

Optimization of qualitative food parameters is an unexplored research topic. There have considerable progress in the optimisation of processed food on quantifiable parameters like nutrition, energy efficiency, processing time, etc [14], [15]. Various cultures and civilisations have honed and revised their cuisines and diet, cooking is still considered an art based on certain heuristics and flexible recipes. This is because the quality of food (typically represented by taste, appearance, texture and smell), is a subjective quantity and the control humans have over the cooking process is limited. Robotic cooking solves the control problem by providing the ability to parameterize the control inputs and execute them with high repeatability. The remaining challenge is the handling of the subjective, noisy, semi-quantitative output data and interpreting the complex relationship between the subjective output and the control inputs [16], [17]. Moreover, as individuals have unique preferences, universal solutions for the optimization problem do not exist. Hence, unlike regular optimization problems, special tools have to be developed for food optimization.

This work presents a robotic platform capable of precisely 
preparing an egg omelette, starting from an uncracked egg all the way till plating the omelette. An industrial robotic manipulator with a custom robotic gripper is used maintaining the generalizability and transferability of the work. The platform has the capability to vary several key parameters for cooking smoothly and consistently. We investigate algorithms for the optimization of subjective food quality measures like taste, appearance and texture [18]. This paper shows how minor changes in the experimental procedure arising out of the optimization framework can lead to richer and cleaner subjective human data and subsequently better food quality. We also show how our method can be used for the bulk optimization of food quality for a group of individuals with distinct preferences.

\section{FOOD QUALITY OPTIMIZATION}

\section{A. Challenges}

Our goal of enhancing food quality is marred by numerous challenges that are unique to this problem. Our proposed optimization solution is designed to address these challenges. 1) Limited function evaluations and fading memory: the cooking process is costly in time and ingredients. The number of samples that can be evaluated by an individual is limited because of finite appetite and the effects of fading memory. Fading memory prevents us from increasing the number of samples by testing over multiple days. 2) Individual preferences: each individual has his/her personal heuristics behind the evaluation of an item. Therefore, optimal solutions obtained for one individual cannot be applied to other individuals. 3) Grounding problem: qualitative evaluations are based relative to the priors of the individual and his/her expectations of the future dataset. For instance, rating of an omelette is the outcome of a complex process resulting from all the previous experiences the user had with omelettes and an implicit idea of the range of omelettes expected from the system.

\section{B. Sequential Bayesian Optimization $v / s$ Batch Bayesian Optimization}

In this work, Bayesian Optimization is used as it is ideal for expensive low-dimensional stochastic functions [19]. The underlying functions are modelled with Gaussian priors which is a suitable fit for the human subjective taste. We investigate two variants of Bayesian Optimization in this paper. The key difference among the two methods is the procedure for obtaining samples for optimization. The first method uses standard Sequential Bayesian Optimization(BO) framework for sequential sampling and optimization. The second method, Batch Bayesian Optimization [20], uses predefined sampling points and Gaussian process models for estimating the expected optimum (See Figure 1). A Gaussian process is fully specified by a mean function $\bar{f}(x)$ and the covariance function $k\left(x, x^{\prime}\right)$

$$
f(x) \sim G P\left(\bar{f}(x), k\left(x, x^{\prime}\right)\right)
$$

Sequential Bayesian Optimization is used for derivativefree global optimization of black-box functions [21]. Based on an initial random sampled data and the update of the priors based on the new observations $\left(Y_{i}\right)$, next query points $\left(X_{i+1}\right)$ are decided. The trade-off between exploration and exploitation is decided by the acquisition function (See Figure 11). The algorithm terminates when the solutions converge based on the stopping criterion. Hence, the number of samples cannot be known a priori. Since the sampling process is dependent on the function output (user feedback), Sequential BO does not allow re-evaluation of previous samples, a problem that exacerbates the grounding problem. Bulk optimization of multiple individuals is not possible because the samples are specific to the individual taste. The tuning of the hyperparameters of the acquisition function is another challenging problem given that we have limited trials and a large control input range.

Batch BO is traditionally used when functions are expensive to evaluate but can be easily parallelized [20]. In our case, it takes time to prepare an omelette but multiple evaluations on the same omelette is not expensive. In Batch Bayesian Optimization, the query points $\left(\left[X_{1}, X_{2} \ldots X_{N}\right]\right)$ are pre-defined and independent from the new observations $\left(\left[Y_{1}, Y_{2} \ldots Y_{N}\right]\right)$. Like, Sequential Bayesian Optimization, Gaussian process models for fitting the prior distributions. The expected maximum $\left(X^{*}\right)$ is then estimated from the fitted probability distribution (see Figure 1 ). The number of samples are fixed in this method. Hence, a termination criterion does not exist in this case. More importantly, now re-evaluation of the samples can be done and user feedback can be modified anytime during the sampling process. This greatly affect the quality of the user feedback for statistical analysis. Additionally, bulk optimization can now be performed.

\section{Experimental Setup}

The experiment is conducted in a customised kitchen as shown in Figure 2. An egg cracker, bowl, electric whisker, salt and pepper, pan, and a whisk (used for mixing while
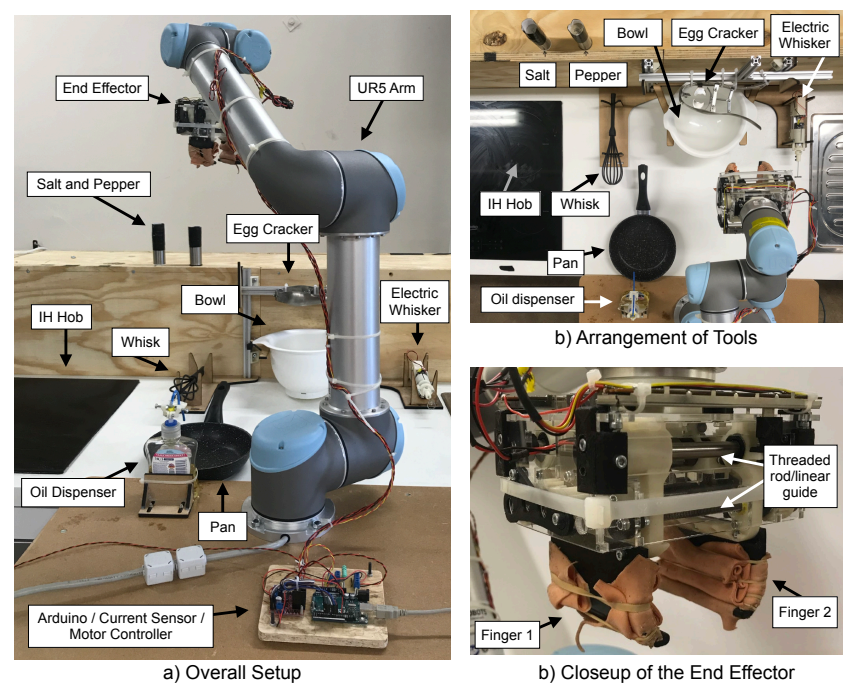

Fig. 2. Experimental setup of the omelette making robot 
heating), are tools that are manipulated by the robot. In order to constrain the tools to a known location, the rig includes holders for the egg cracker, bowl, electric whisker, oil dispenser, and the whisk. Similarly, the initial location for the salt and pepper dispensers are marked.

Figure 3 shows a block diagram of the robot system. A UR5 robot arm from Universal Robotics is used as the manipulator for the omelette cooking process. The UR5 is controlled by a python script using a provided API to communicate with the UR5 control box. Through the API, commands to the UR5 such as, Cartesian coordinate position demands, individual joint angle demands, and force control can be sent.

A simple end-effector has been designed and manufactured to manipulate all the tools in the kitchen setup. It has two fingers that can move parallel to each other independently via two DC motors. The fingers are equipped with silicone padding better grasp tools with varying shapes. A linear potentiometer is connected to each finger which is used for position feedback and to avoid collisions. The current input to the motor controller is read from a shunt resistor, which effectively indicates the grasping force. This feedback is used to tune the strength of the grip when an object is sensed. An Arduino Uno was used to read the sensors on the gripper and control the motor through a dual H-bridge circuit. Serial communication allows the Arduino to communicate with the python script controlling the UR5, to coordinate the grasping and arm movements.

\section{ROBOTIC COOKING}

\section{A. Omelette Cooking Procedure}

The omelette cooking procedure is illustrated in Figure 4. where the robot moves through a sequence of events. The robot is reliant on all the tools setup in the known location to initiate the process, but can accommodate slight changes to the tool placement via the gripper robustness and methods such as force feedback when grasping/using the tools. The eggs are placed in the cracker manually. On developing the experimental setup and the sequence for omelette cooking, a list of vital control parameters were identified as shown in Table [1. The heat-level of the hob, the time from placing the pan to heating, and the time from pouring the contents from the bowl until mixing are kept constant.

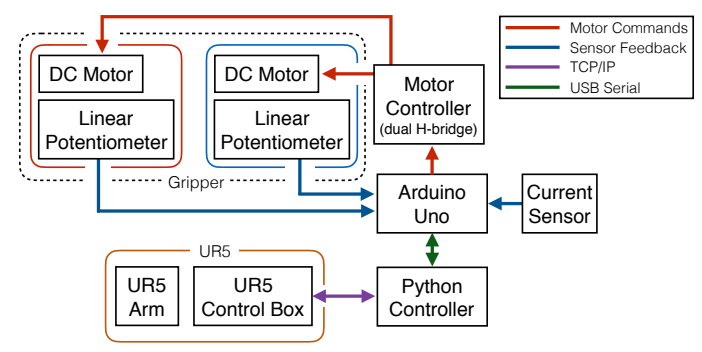

Fig. 3. Electrical and control architecture

\section{METHODS}

\section{A. Investigation of Control Parameters}

An investigation of the control parameters was conducted to better understand their relationship with the user feedback. With the aim of dividing the multi-objective optimization problem into groups of multiple single-objective optimization problem, a sensitivity test was performed initially. In this test, four human volunteers are first given a base omelette prepared with control inputs set to their middle. Subsequently, omelettes with one of the input parameters increased to its maximum value while keeping the others constant, are prepared and evaluated. The human subjects are informed that the first omelette will be a baseline to be compared against, but are not informed of the input parameters. Accounting for effect of fading memory, each control parameter is only varied once for our analysis.

The sensitivity of an output for a particular input is calculated average relative change of the output (when the input in question has been modified) with respect to the base omelette, given as such.

$$
\operatorname{Sensitivity}\left(Y_{j} \mid X_{i}\right)=\sum_{n=1}^{N} a b s\left(\frac{Y_{n, j}-Y_{\text {base }}}{\sigma\left(Y_{n}\right)}\right)
$$

Here, $Y_{n, j}$, refers to the feedback on the variable $j$ by user $n, X_{i}$ is the corresponding control parameter, $Y_{\text {base }}$ is the base feedback value and $\sigma$ represents the standard deviation of the sampled variables.

The results obtained from this analysis are shown in Figure 5. Based on this analysis, functional dependencies are formulated based on the three most influential control parameters. Salt, pepper, and mixing were found to affect the taste metric, flavour, the most. The three control parameters that affected appearance and texture were mixing, whisking and cook-time. Hence, we reformulated the problem as two separate optimization problems; one for flavour and another for the combined value of appearance and texture. The grouping has not been disclosed to any human volunteers across the experiments.

\section{B. User Feedback and Optimization}

The human assessor evaluates a sample after the omelette is prepared on three food taste metrics: Flavour, Appearance,

TABLE I

INPUT/OUTPUT VARIABLES

\begin{tabular}{l}
\begin{tabular}{|c|c|c|c|c|}
\hline Control Input X & Quantity & Units & Min & Max \\
\hline \hline s & Salt & Shakes & 0 & 10 \\
\hline p & Pepper & Shakes & 0 & 10 \\
\hline $\mathrm{m}$ & Mixing & Rotations on pan & 0 & 10 \\
\hline $\mathrm{w}$ & Whisking & Seconds & 0 & 12 \\
\hline $\mathrm{c}$ & Cook Time & Seconds & 210 & 510 \\
\hline User Feedback Y & Quantity & Expected Outcome \\
\hline \hline f & Flavour & $0-10$ \\
\hline a & Appearance & $0-10$ \\
\hline t & Texture & -10 \\
\hline
\end{tabular} \\
\hline
\end{tabular}




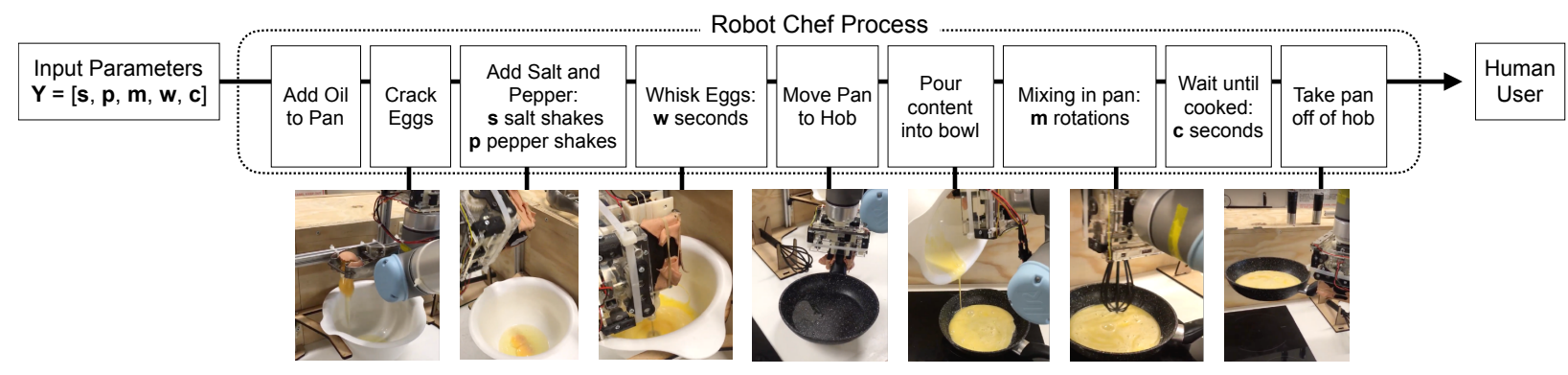

Fig. 4. Procedure of omelette cooking by the robotic chef

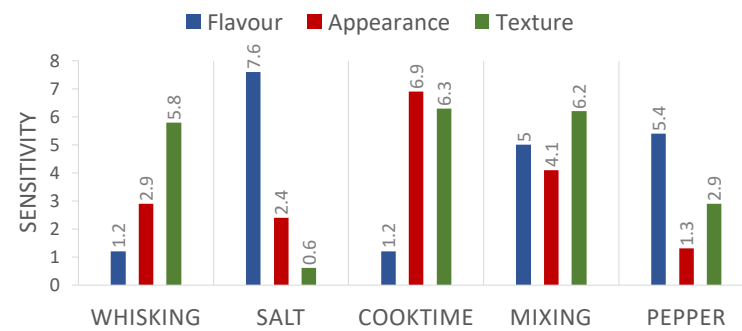

Fig. 5. Sensitivity of the food evaluation metrics to the control variables. A higher sensitivity indicates higher influence of the control input on their corresponding metric.

and Texture (Table I). The other commonly used metric, Aroma, is ignored for our studies due to its weak dependence on our control parameters. For the three taste metrics, the assessor will score the omelette sample within a range of 0 to 10 (only integer values) independently.

The BayesianOptimization library for python3 was been used to perform the optimization process (https://github.com/fmfn/BayesianOptimization). For all the Sequential Bayesian Optimization tests, two optimization routines were run in parallel, one for flavour and the other for appearance and taste. The Gaussian Process (GP) employed a Matern kernel $(n u=2.5)$. The only parameter we tune for the optimization procedure is the exploration parameter $(\kappa)$ of the acquisition function based on the GP Upper Confidence Bound. Four experiments were conducted to optimize the output of four individuals, with varying levels of exploration (Table III). Higher exploration leads to a higher probability of finding the global optima but with slower convergence. In our case, we have an upper limit on the number of iterations; due to the finite number of samples an individual can taste and the fading memory effect. Therefore, it is impossible to ensure a global optima. By the nature of this method, the volunteer was not allowed to change the evaluation of the omelettes after a score has been given, but is allowed to view/taste the previous omelettes if necessary.

For the Batch Bayesian Optimization a GP regression model was used with automatic relevance determination (ARD) squared exponential kernel function. There were no parameters to be tuned for this case. A range of control parameters $\left(X_{\text {Total }}=\left[X_{1}, X_{2} \ldots X_{10}\right]\right)$ were selected and the
TABLE II

KAPPA HYPERPARAMETER USED IN SEQUENTIAL BO

\begin{tabular}{|l|l|l|}
\hline Experiment & $\begin{array}{l}\text { Kappa } \\
\text { (Flavour) }\end{array}$ & $\begin{array}{l}\text { Kappa } \\
\text { (Appearance) }\end{array}$ \\
\hline 1 & 3 & 0.6 \\
\hline 2 & 3 & 0.6 \\
\hline 3 & 1 & 1 \\
\hline 4 & 1.2 & 1.2 \\
\hline
\end{tabular}

10 omelettes corresponding to each input $Y_{i}$ were evaluated, resulting in output feedback $\left(Y_{\text {Total }}=\left[Y_{1}, Y_{2} \ldots Y_{10}\right]\right) . Y_{\text {Total }}$ and $X_{\text {Total }}$ is then fed into the Batch Bayesian Optimizer to return the optimal set of input parameters, $X^{*}$. Two experiments were conducted with 4 volunteers. The 10 input parameter sets were obtained by a constrained random process. In the two search spaces, (Salt, Pepper, Mixing) and (Mixing, Whisking, Cooktime), corresponding to the two output groups, each input was required to be as far away as possible (euclidean distance) from every other input set whilst the Mixing parameter consistent between the two. Subjects evaluating the sample omelettes were allowed to retract and change their evaluation of the omelettes throughout the experiment, but were left unaware of the optimization process and the objective of the study throughout.

Two methods were investigated to obtain the optimized control parameters, $X^{*}$. The first method was performing two independent Batch Bayesian Optimization's on the "Flavour" output and the "Appearance and Texture" output separately. The second method groups all of the output into one value by summing the mean normalised output values from "Flavour", "Appearance" and "Texture". Two optimized omelettes are produced corresponding to the two optimization methods to be tested with the subject.

\section{RESULTS}

\section{A. Demonstration of Omelette Making}

A total of 6 experiments were conducted on various days to test the experimental setup and our optimization algorithms. Four of the tests were for the Sequential BO and the remaining two were conducted for the Batch BO. As Batch BO allows bulk optimization, the final test was done with three assessors and optimized products for each volunteer was provided from the common sampling process (Table III). Note that experiment number 2 had four assessors, 
but the optimization was performed for a randomly selected individual. A total of 73 omelettes were prepared for the optimization study. Some samples prepared by the robotic chef are shown in Figure 6 Omelettes prepared using the same input parameters had good repeatability as shown by the visual similarity through the images of the omelettes and the RGB histogram in Figure 6. The robotic platform is critical to obtain repeatable samples while maintaining its generalizability to cook various recipes, which is not achievable by humans or with an automated machine. The robotic manipulator is used to make our approach general. For this, we have been effortful to use available kitchen appliances and utensils.

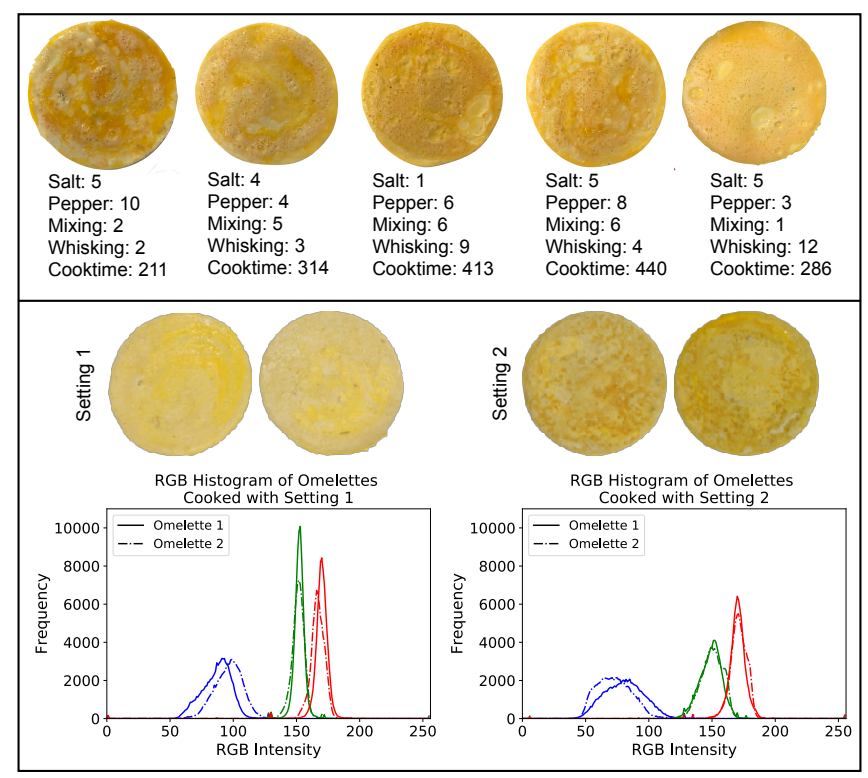

Fig. 6. Above: various type of Omelettes prepared by the robotic chef. Below: repeatability of the samples achievable due to the robotic platform (shown here by the visual similarity of same input Omelettes)

TABLE III

QUANTITY OF EXPERIMENTS AND OMELETTES

\begin{tabular}{|l|l|l|l|l|}
\hline \multirow{4}{*}{ Sequential } & $\begin{array}{l}\text { Experiment } \\
\text { Number }\end{array}$ & $\begin{array}{l}\text { Omelettes } \\
\text { Prepared }\end{array}$ & $\begin{array}{l}\text { Optimized } \\
\text { Assessors }\end{array}$ & $\begin{array}{l}\text { Number of } \\
\text { Assessors }\end{array}$ \\
\hline & 1 & 15 & 1 & 1 \\
\cline { 2 - 5 } & 2 & 10 & 1 & 4 \\
\cline { 2 - 5 } & 3 & 10 & 1 & 1 \\
\cline { 2 - 5 } & 4 & 10 & 1 & 2 \\
\hline \multirow{3}{*}{ Batch } & 5 & 12 & 1 & 1 \\
\cline { 2 - 5 } & 6 & 16 & 3 & 3 \\
\hline
\end{tabular}

\section{B. Results of Food Optimization}

The main advantage of the Batch $\mathrm{BO}$ is the ability to efficiently explore the input space without fear of nonconvergence. Combined with the flexibility it provides the assessors for re-evaluation of the omelettes, Batch BO leads to richer user feedback for the final optimization. As the Sequential BO was tuned to achieve convergence, the exploration of the input space was low. Whereas Batch BO

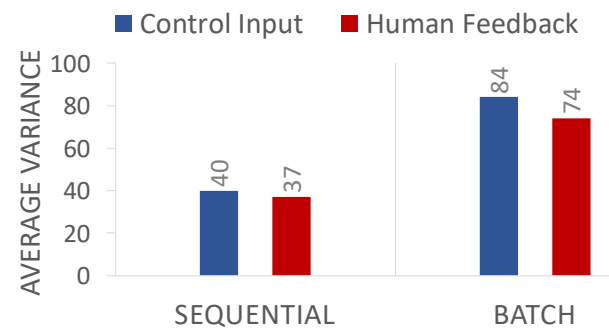

Fig. 7. Average variance of control inputs and the human feedback during the sampling process. Higher variance during the exploration of the control variables leads to higher precision and reliability of the qualitative feedback.

achieved higher exploration due to the near uniform distribution of the input parameters. When Sequential BO tuned for higher exploration, it was difficult to achieve convergence within the required iterations. This can be seen in Figure 7. The variance of both the input parameters and feedback are both more than twice for Batch BO compared to Sequential BO. This shows that in Batch BO 1) a higher level of exploration has been performed, and 2) the human assessor experienced a higher variability in the omelettes. Both these aspects relate to the 'reliability' of the experiment. With little exploration, it is more difficult to conclude if an optimum has been reached. With low variability in the output feedback, the effect of noise is likely to be higher. An example of the sampling process and the user feedback is shown in Figure 8. Notice the lower ranges in the output feedback in the Sequential BO when compared to the Batch BO. Another interesting noise artefact that arises with low control input variance is the unconscious effect of 'output merging'. This can be observed vividly towards the final few samples in the Sequential BO experiment when the three output feedback seems to be directly correlated to each other. This effect could possibly be reduced further by increasing the input variance progressively over iterations.

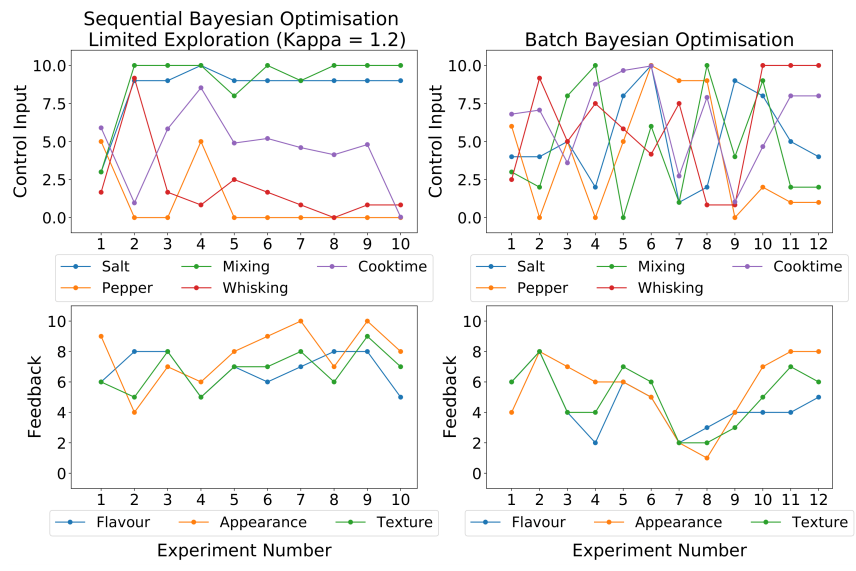

Fig. 8. Sequential BO has a trade-off between exploration of the input space and number of iterations for convergence. Hence, there is insufficient input variance (exploration) as shown in this case. Batch BO is immune to this trade-off.

Figure 9 complements the result of Figure 7 by showing the relative improvements in subjective food quality using 
the two optimization methods for all the 8 assessors. We do not have sufficient data points to provide individual analysis and hence all our results are ascertained from the population data. The relative improvement(R.I) measures the subjective improvement of optimization procedure. It is measured by the change in the subjective feedback score with respect to the mean score during sampling scaled the variance of the control parameters after the end of the optimization cycle. The scaling of scores by variance is important to indicate the confidence of the measured subjective change. For instance, an observed improvement in qualitative rating for a constant input can be attributed to the noise in the system and hence of no relevance.

$$
\begin{gathered}
R . I_{n, j}= \\
\left\{\frac{Y_{n, K, j}+Y_{n, K-1, j}}{2}-\frac{1}{K} \sum_{k=0}^{K} Y_{n, k, j}\right\} \times \sum_{i} \sigma^{2}\left(n, X_{i}\right)
\end{gathered}
$$

where $k$ represents omelettes assessed.

Compared to sequential $\mathrm{BO}$, the expected improvement in performance of the Batch $\mathrm{BO}$ arise because of the selfregulating effects of higher exploration and re-evaluation. Higher discernability among samples leads to better 'grounding' of the subjective data and hence better fitting of the underlying objective function.

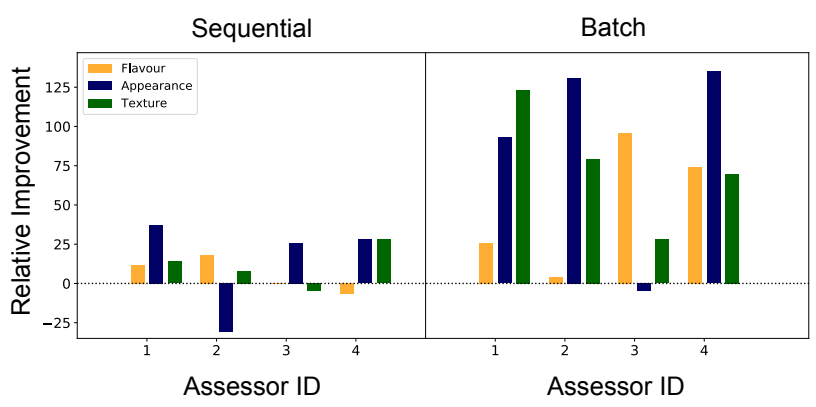

Fig. 9. Relative food quality improvement with the two optimization methods

\section{CONCLUSIONS}

This work introduces the concept of robotic food quality optimization and present an efficient and reliable optimization routine for enhancement of food taste. We present a robotic platform that can cook parameterizable and accurate omelettes. The platform uses general kitchen appliances, a common industrial manipulator and a custom simple gripper for the cooking process in consideration of extending the methodology to other dishes. Our optimization solution is addressed to solve the main challenges of qualitative data (See Section II-A). We present the standard Sequential Bayesian Optimization method which is particularly suited for expensive, noisy low-dimensional cost functions and its variant, the Batch Bayesian Optimization as a better extension. The results indicate that quantifiable improvements can be obtained by this methodology for food optimization.
Additionally, such an approach can be extended easily for bulk optimization and even parallel optimization using multiple robotic chefs. Further studies have to be conducted to investigate other optimization techniques and their viability.

Handling subjective data is difficult. With enough sample data and proper statistical techniques, subjective data can be reasonably quantified. The food optimization problem is however severely restricted by the sample availability and will always be susceptible to subjective elements. This exactly why the problem is interesting and relevant for human-robot interaction. To reduce the subjectiveness of the data, we have always employed normalization techniques for quantitative comparisons, which is still one of the common approaches for qualitative data analysis. We believe that this work will become more relevant in the future for HumanRobot Interaction tasks, which typically involves qualitative feedback along with quantitative feedback.

Food optimization is not limited to qualitative feedback. Other methods that rely on quantitative data can also be another direction to investigate. A straightforward problem that can be easily solved is to use the same methodology for optimizing food nutrient and calorie content. With the ability to personalize diet using data from past and information from other users, this could revolutionize the way we prepare our food and the design of our kitchens. There are also other methods to obtain taste feedback that are 'more' quantitative, like measurement of neural activity during the process of eating [22]. Another method could be to use vision-based feedback for appearance optimization. The problem of qualitative data analysis is not only restricted to food quality optimization, but also relevant for seamless integration of robotic assistants in various domestic tasks.

\section{ACKNOWLEDGMENT}

This work was supported by BEKO PLC and Symphony Kitchens. We especially thank the valuable input and discussion with Dr Graham Anderson and Dr Natasha Conway.

\section{REFERENCES}

[1] C. C. Kemp, A. Edsinger, and E. Torres-Jara, "Challenges for robot manipulation in human environments [grand challenges of robotics]," IEEE Robotics \& Automation Magazine, vol. 14, no. 1, pp. 20-29, 2007.

[2] C.-J. Du and D.-W. Sun, "Learning techniques used in computer vision for food quality evaluation: a review," Journal of food engineering, vol. 72, no. 1, pp. 39-55, 2006.

[3] L. Scimeca, P. Maiolino, D. Cardin-Catalan, A. P. del Pobil, A. Morales, and F. Iida, "Non-destructive robotic assessment of mango ripeness via multi-point soft haptics," in 2019 International Conference on Robotics and Automation (ICRA). IEEE, 2019, pp. $1821-1826$

[4] C.-J. Du and D.-W. Sun, "Recent developments in the applications of image processing techniques for food quality evaluation," Trends in food science \& technology, vol. 15, no. 5, pp. 230-249, 2004.

[5] S. V. Ilyukhin, T. A. Haley, and R. K. Singh, "A survey of automation practices in the food industry," Food Control, vol. 12, no. 5, pp. 285296, 2001.

[6] J. Hughes, L. Scimeca, I. Ifrim, P. Maiolino, and F. Iida, "Achieving robotically peeled lettuce," IEEE Robotics and Automation Letters, vol. 3, no. 4, pp. 4337-4342, 2018.

[7] P. Y. Chua, T. Ilschner, and D. G. Caldwell, "Robotic manipulation of food products-a review," Industrial Robot: An International Journal, vol. 30, no. 4, pp. 345-354, 2003. 
[8] M. Bollini, J. Barry, and D. Rus, "Bakebot: Baking cookies with the pr2," in The PR2 workshop: results, challenges and lessons learned in advancing robots with a common platform, IROS, 2011.

[9] M. Bollini, S. Tellex, T. Thompson, N. Roy, and D. Rus, "Interpreting and executing recipes with a cooking robot," in Experimental Robotics. Springer, 2013, pp. 481-495.

[10] M. Beetz, U. Klank, I. Kresse, A. Maldonado, L. Mösenlechner, D. Pangercic, T. Rühr, and M. Tenorth, "Robotic roommates making pancakes," in 2011 11th IEEE-RAS International Conference on Humanoid Robots. IEEE, 2011, pp. 529-536.

[11] A. Petit, V. Lippiello, G. A. Fontanelli, and B. Siciliano, "Tracking elastic deformable objects with an rgb-d sensor for a pizza chef robot," Robotics and Autonomous Systems, vol. 88, pp. 187-201, 2017.

[12] J. Sun, W. Zhou, D. Huang, J. Y. Fuh, and G. S. Hong, "An overview of $3 \mathrm{~d}$ printing technologies for food fabrication," Food and bioprocess technology, vol. 8, no. 8, pp. 1605-1615, 2015.

[13] J. I. Lipton, "Printable food: the technology and its application in human health," Current opinion in biotechnology, vol. 44, pp. 198201, 2017.

[14] J. R. Banga, E. Balsa-Canto, C. G. Moles, and A. A. Alonso, "Improving food processing using modern optimization methods," Trends in food science \& Technology, vol. 14, no. 4, pp. 131-144, 2003.

[15] N. Perrot, I.-C. Trelea, C. Baudrit, G. Trystram, and P. Bourgine, "Modelling and analysis of complex food systems: state of the art and new trends," Trends in Food Science \& Technology, vol. 22, no. 6, pp. 304-314, 2011.

[16] J. E. Harris, P. M. Gleason, P. M. Sheean, C. Boushey, J. A. Beto, and B. Bruemmer, "An introduction to qualitative research for food and nutrition professionals," Journal of the American Dietetic Association, vol. 109, no. 1, pp. 80-90, 2009.

[17] E. Lutton, N. Perrot, and A. Tonda, Evolutionary Algorithms for Food Science and Technology. Wiley Online Library, 2016, vol. 7.

[18] M. Pelikan, D. E. Goldberg, and E. Cantú-Paz, "Boa: The bayesian optimization algorithm," in Proceedings of the 1st Annual Conference on Genetic and Evolutionary Computation-Volume 1. Morgan Kaufmann Publishers Inc., 1999, pp. 525-532.

[19] P. I. Frazier, "A tutorial on bayesian optimization," arXiv preprint arXiv:1807.02811, 2018.

[20] J. Azimi, A. Fern, and X. Z. Fern, "Batch bayesian optimization via simulation matching," in Advances in Neural Information Processing Systems, 2010, pp. 109-117.

[21] K. A. Saar, F. Giardina, and F. Iida, "Model-free design optimization of a hopping robot and its comparison with a human designer," IEEE Robotics and Automation Letters, vol. 3, no. 2, pp. 1245-1251, 2018.

[22] G. Borg, H. Diamant, L. Ström, and Y. Zotterman, "The relation between neural and perceptual intensity: A comparative study on the neural and psychophysical response to taste stimuli," The Journal of physiology, vol. 192, no. 1, pp. 13-20, 1967. 